\section{Comment on "Global $\boldsymbol{N}$-Acetylaspartate Declines Even in} Benign Multiple Sclerosis"

We have read with great interest the article "Global N-Acetylaspartate Declines Even in Benign Multiple Sclerosis" by Rigotti et al. ${ }^{1}$ It is generally accepted that axonal injury is the pathologic substrate for the symptoms and disability seen in patients with multiple sclerosis (MS), a supposed inflammatory central nervous system disease of unknown origin. The visualization of certain metabolites such as $\mathrm{N}$ acetylaspartate (NAA) may represent axonal loss, axonal integrity, or axonal function.

It remains completely unclear why each patient with MS follows his or her own individual disease course and why, until now, no marker could be established that may allow a reliable prediction. ${ }^{2}$ NAA levels vary even in healthy controls and decline with age. A supposed NAA decline per year, extrapolated by a mathematic model including only 1 NAA baseline measurement, disease duration, and so forth, could never replace a long-term follow-up study (as already mentioned by the authors). However, it is still very intensely discussed whether axonal injury does really exist in all patients with MS and, if so, whether axonal injury already occurs in the very early disease stages and progresses steadily and "clinically silently," even when patients with MS remain clinically stable. That the NAA levels of 43 patients with relapsing-remitting MS (RRMS) with very benign dis- ease courses, very low lesion load, and, in part, very long disease duration (range, 15-35 years) are that low is astonishing and should have been discussed in detail and compared with the different data published recently. ${ }^{3-6}$ Did the included patients with RRMS fulfill the Barkhof criteria and were oligoclonal bands detectable?

\section{References}

1. Rigotti DJ, Gonen O, Grossman RI, et al. Global $N$-acetylaspartate declines even in benign multiple sclerosis. AJNR Am J Neuroradiol 2011;32:204-09

2. Gilmore CP, Cottrell DA, Scolding NJ, et al. A window of opportunity for no treatment in early multiple sclerosis? Mult Scler 2010;16:756-59

3. Benedetti B, Rovaris M, Rocca MA, et al. In vivo evidence for stable neuroaxonal damage in the brain of patients with benign multiple sclerosis. Mult Scler 2009;15:789-94

4. Kirov II, Patil V, Babb JS, et al. MR spectroscopy indicates diffuse multiple sclerosis activity during remission. J Neurol Neurosurg Psychiatry 2009;80: 1330-36

5. Aboul-Enein F, Krssák M, Höftberger R, et al. Reduced NAA levels in the NAWM of patients with MS is a feature of progression: a study with quantitative magnetic resonance spectroscopy at 3 Tesla. PLoS One 2010;5:e11625

6. Aboul-Enein F, Krssák M, Höftberger R, et al. Diffuse white matter damage is absent in neuromyelitis optica. AJNR Am J Neuroradiol 2010;31:76-79

F. Aboul-Enein

Department of Neurology

SMZ-Ost Donauspital

Vienna, Austria

DOI 10.3174/ajnr.A2528 